\title{
API de Google Maps para un mapa de conocimiento de los asesores especializados de un Centro de Desarrollo Empresarial
}

\author{
José Sergio Ruiz Castilla, José Antonio Díaz García, Jair Cervantes Canales \\ Centro Universitario UAEM Texcoco, \\ Universidad Autónoma del Estado de México, \\ Estado de México, México \\ jsergioruizc@gmail.com, ittonzs@gmail.com, chazarra17@gmail.com
}

\begin{abstract}
Resumen. Existen sitios dedicados a asesorar a emprendedores y micro empresarios en temas de compras, producción, innovación, ventas, finanzas, etc. Tanto en México, como en otros países, existen los CDE (Centros de Desarrollo Empresarial) otros denominados Incubadoras de Empresas, donde se brinda apoyo a los emprendedores que inician una nueva empresa. En la Universidad Autónoma del Estado de México, se creó una red de Incubadoras de Empresas en los Centros Universitarios, Una de estas unidades se encuentra en el Centro Universitario UAEM Texcoco, donde se enfocó este proyecto. Una de las misiones de los CDE es ofrecer y llevar a cabo asesorías ante los problemas y retos de las empresas nacientes. Para lo anterior se creó una aplicación Web para administrar el conocimiento a través de un Mapa de Conocimiento. Los usuarios pueden consultar la existencia de conocimiento requerido; si existe, lo podrán visualizar en un mapa con ayuda de la API (Interfaz de Programación de Aplicaciones) de Google Maps. Para su uso, basta un dispositivo con conexión a internet. Esta aplicación busca apoyar a emprendedores y empresarios que tiene las preguntas: ¿El CDE Tiene el conocimiento qué requiero? ¿Quién tiene el conocimiento? ¿Dónde están los portadores del conocimiento? ¿Cómo y dónde los contacto? De existir el conocimiento en el mapa bastará contactar a algún portador y establecer una modalidad para la asesoría requerida. Se parte, de la búsqueda de conocimiento, su existencia, ubicación y forma de contactar al portador del conocimiento. Para lo anterior se logró una aplicación capaz de filtrar un tipo de conocimiento y el mapeo sobre la plataforma de la API de Google Maps.
\end{abstract}

Palabras clave: Conocimiento, mapa de conocimiento, Google Maps, emprendedores, asesoría especializada. 


\section{Introducción}

Las IE (Incubadoras de Empresas) y CDE tiene la misión de apoyar a emprendedores y microempresarios que desean que sus empresas tengan crecimiento o mejora de utilidades, procesos, calidad u otros propósitos.

Es preciso contar con expertos que una vez registrados, forman parte de una red de AE (Asesores Especializados). De cada AE se conoce su nombre o razón social, alguna dirección o solo ubicación, así como forma de contacto: correo electrónico, página Web, teléfono celular o fijo u otro medio que establezca.

Una vez registrada la red de AE; se requiere un IC (Inventario de Conocimientos). El IC contiene los conocimientos de los AE que puede ser actualizado en medida que se adquieren nuevos conocimientos.

Para la administración del conocimiento se define un catálogo de conocimientos, que a través de una base de datos, es posible su almacenamiento y consulta.

Por otro lado la tecnología nos permite otras nuevas formas de consultar la información y la comunicación entre personas e instituciones. Con un simple clic, se puede navegar por el mundo sin siquiera moverse de la oficina, accediendo a las bases de datos de algunos servidores que existen en el planeta.

Ya existen sitios capaces de buscar una ubicación, una ruta, un taxi, un restaurante, un café, museos. ¿Por qué no, sitios, para buscar conocimiento?

Este sistema permite conocer: ¿qué conocimiento existe?, ¿quién lo posee?, ¿dónde están los portadores? y ¿cómo acceder al conocimiento?

La API (Interfaz de Programación de Aplicaciones) de Google Maps ${ }^{1}$ puede incorporarse a las aplicaciones ofreciendo mostrar vistas de calles o satélite. Además es posible: insertar ubicaciones e información personalizada. Así se muestra en los resultados.

\section{El conocimiento}

La historia de la GC (Gestión del Conocimiento) ha venido evolucionando hasta el punto que aún no se encuentra una definición clara y completa a este término; a lo que muchos autores han tratado de formular su propia definición, como Nonaka y Takeuchi; planteando que "La Gestión del Conocimiento implica llevar los conocimientos correctos a las personas que lo necesitan "just in time" con el objeto que puedan resolver el problema que deseen con prontitud y eficacia" [7].

Otra definición sería la de "Tratar de extraer lo mejor de las personas de la organización utilizando sistemas que permiten que la información disponible se convierta en conocimiento" [1].

Se puede afirmar que al gestionar el conocimiento facilita:

- La localización de fuentes de conocimiento.

- La reutilización de experiencias.

1 Google Maps en una aplicación de la Empresa Google Inc. Sistema de mapas digitales lanzado en febrero de 2005. Disponible en www.maps.google.com. 
- La mejora de los procesos de negocios.

- La reutilización de artefactos del proceso.

Existiendo experiencia en organizaciones que demuestran que se puede gestionar el conocimiento y se empiezan a adoptar arquitecturas Fig. 1 para dicha gestión, donde éstas, se puede considerar como el nexo que une a las actividades de producción diarias con las actividades de mejora y los objetos de negocio.

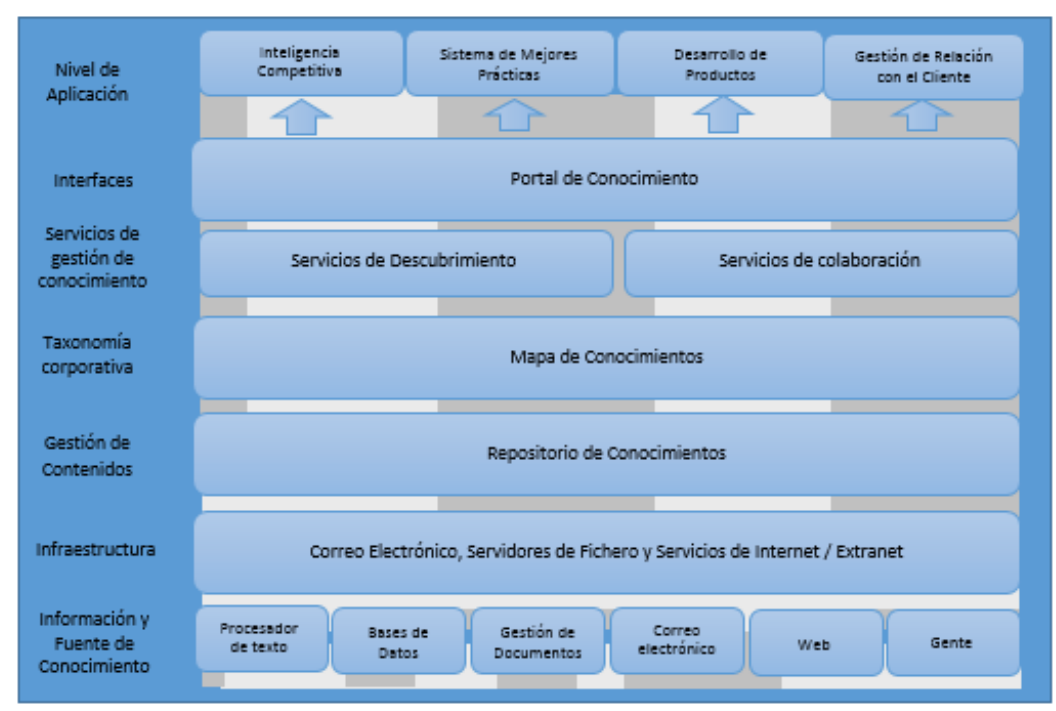

Fig. 1. Arquitectura de gestión de conocimiento (Piattini, 2007).

\subsection{Asesores de negocios}

Un asesor de negocios es un consultor que ofrece a los propietarios de empresas conocimiento que les ayude a manejar sus negocios más eficazmente. Estos pueden ser consultados para ayudar a simplificar o mejorar un negocio, o pueden jugar un papel más activo, que ofrece sesiones de asesoramiento recurrente a sus clientes.

Estos asesores pueden ayudar a diagnosticar dónde y por qué una empresa tiene mal desempeño, para revertir una tendencia negativa, además de ayudar a las personas con el desarrollo de un nuevo negocio o una nueva diversificación en un negocio existente.

\subsection{Centro de Desarrollo Empresarial}

Es un proceso dinámico para la estimulación de nuevos y pequeños proyectos empresariales. Por ellos las incubadoras otorgan a las empresas y proyectos jóvenes, dentro del campo competitivo, apoyo durante sus etapas de desarrollo, ya que en estas son más vulnerables. 


\subsection{Historia de la Incubadora de empresas}

La existencia de incubadoras de empresas nació hace casi 50 años en los Estados Unidos.

La gran mayoría de las empresas que inician, no llegan a desarrollarse, y es aquí donde apoyan las incubadoras, ya que ayuda a reducir la mortalidad de dichas empresas.

La Asociación Mexicana de Centros para el Desarrollo de la Pequeña Empresa surge ante la necesidad de contar con un organismo que apoye a los centros de desarrollo de las PyMEs que han surgido en nuestro país, en sus esfuerzos para el desarrollo de la pequeña empresa, representando el interés colectivo de dichos centros ante entidades gubernamentales y privadas, nacionales o extranjeras [2].

\subsection{Atención a emprendedores}

A través de dos departamentos, el de incubación de empresas y el de capacitación empresarial, el CDE, brinda a los nuevos emprendedores servicios indispensables para ellos, como son: tutoría y asesoría, en donde se desarrollan las propuestas de mejora, comercial, técnica, financiera y administrativa para empresas en operación.

En el desarrollo de negocios, se hace un acompañamiento y soporte del emprendedor a través de las etapas de diagnóstico, gestación e incubación de un proyecto.

El albergue, donde en los primeros meses de operación, el emprendedor/empresario podrá contar con el uso de un espacio de oficina con sus servicios administrativos necesarios [3].

\subsection{Google Maps}

Es una aplicación de Google Inc. Es un servidor de aplicaciones de mapas en la Web que ofrece imágenes de mapas desplazables, así como fotos satelitales del mundo, e incluso la ruta entre diferentes ubicaciones o imágenes a pie (utilizando la API de Google Street View) ${ }^{2}$.

Es idéntico a Google Earth $^{3}$, una aplicación que ofrece vistas del globo terráqueo, sea de día o de noche, pero que no es fácil de integrar a páginas Web. Está disponible para Android ${ }^{4}$ y Java $\mathrm{ME}^{5}$.

Google Maps ofrece la capacidad de hacer acercamientos o alejamientos para mostrar el mapa. El usuario puede controlar el mapa con el mouse o las teclas de

2 Aplicación de Google Inc. Que permite visualizar calles de las principales ciudades, como si lo hiciera a pie.

3 Aplicación de Google Inc. Permite ver la tierra en forma de mapa de carreteras e imágenes satelitales.

4 Sistema Operativo creado por Google Inc. Actualmente integrado en la mayoría de tabletas y Smartphones.

5 Plataforma para el lenguaje de Java bajo la dirección de la Empresa Sun Microsystems. Disponible en: http://www.oracle.com/us/sun/index.htm. 
dirección para moverse a la ubicación que se desee. Los usuarios pueden ingresar una dirección, una intersección o un área en general para buscar en el mapa. Los resultados de la búsqueda pueden ser restringidos a una zona, gracias a Google Local. Por ejemplo, si alguien quiere consultar por "Waffles in Ottawa", para encontrar restaurantes que sirven este alimento cerca de la ciudad. Las búsquedas pueden encontrar una amplia gama de restaurantes, hoteles, teatros y negocios generales.

Las coordenadas de Google Maps se encuentran en el sistema WGS84 ${ }^{6}$, el cual mostrará la latitud y la longitud, positiva para Norte y Este, negativa para Sur y Oeste. Hay varias formas de obtenerlas, una vez que se ha localizado el lugar que nos interesa.

\subsection{Actividad de compartir el conocimiento en México.}

Las lecciones aprendidas se obtienen al culminar una tarea, actividad o un proyecto, las cuales se deben documentar para una consulta posterior por todos los integrantes de la organización [12, 13]. En México el modelo MoProSoft (Modelo de procesos para el desarrollo de software) recomienda almacenar la documentación de los procesos en una base de conocimiento de acceso libre para los integrantes de la organización [13].

Compartir el conocimiento en las organizaciones es una ventaja competitiva que permite agregar valor a los productos y servicios y a la organización misma. Sin embargo para compartir el conocimiento es necesario un ambiente, herramientas y procesos definidos para dicha tarea [4].

El conocimiento se genera y se acumula a partir de lo que sé, de lo que aprendo de otros y lo que aprendo haciendo. Una vez que se posee se debe compartir con los demás logrando un conocimiento colectivo u organizacional [15].

Compartir el conocimiento de persona a persona requiere de un ambiente adecuado, pero en organizaciones distribuidas o grandes es necesarias herramientas donde es necesario trasformar el conocimiento tácito a explícito y de explícito a tácito $^{7}[7]$.

\subsection{Transferencia del conocimiento en México}

En México existe transferencia de conocimiento; pero, ¿cómo se transfiere el conocimiento? podría ser cara a cara, con cursos de capacitación o desde internet; sin embargo ¿cómo lo hacen? Podría ser informalmente, porque no existen procesos y políticas que lo incluyan como actividad en la creación de nuevas empresas.

6 Es un sistema de coordenadas geográficas mundial que permite localizar cualquier punto de la Tierra (sin necesitar otro de referencia) por medio de tres unidades dadas. WGS84 son las siglas en inglés de World Geodetic System 84 (que significa Sistema Geodésico Mundial 1984).

${ }^{7}$ Se considera la obra de Nonaka y Takeuchi, a pesar de su antigüedad, por ser la obra clásica que fundamenta el conocimiento tácito y explícito así como el proceso de la transferencia del conocimiento; que a pesar del año de publicación sigue siendo vigente. 
Otra forma es a través de textos, repositorios de documentos, la intranet, groupware y SGC (Sistema de Gestión del conocimiento). Y una forma más consiste en aprender viendo, se dispone de un experto y un aprendiz que al observar cómo se desarrollan las actividades aprende [5, 6].

La socialización es el medio más eficaz para la transferencia del conocimiento tácito-tácito, por eso en Japón se acostumbra que las personas socialicen, de hecho prefieren la transferencia del conocimiento tácito [11].

\subsection{Almacenamiento del conocimiento}

Es importante obtener un inventario de activos de conocimiento, que corresponden al conjunto de conocimientos, que posee una persona o plasmado en un medio y que guarda de forma cohesiva la solución de un problema específico, dentro de un dominio del conocimiento.

En la empresa se guardan todos los activos de conocimiento explícito en una base de conocimiento; dichos activos de conocimiento explícito aumentarán en medida que se introduzcan a la base de conocimiento.

\subsection{Inventario del conocimiento}

Para el almacenamiento del conocimiento es necesario un repositorio de conocimiento denominado base de conocimiento donde se almacenan el conocimiento que poseen los AE. El conocimiento almacenado forma parte de un inventario de conocimiento. La unidad son componentes de conocimientos llamados activos de conocimiento $[5,10]$.

\section{Método utilizado}

\subsection{Identificación de los Asesores Especializados}

Los AE son docentes o personal de apoyo que se encuentran dentro o fuera del Centro Universitario UAEM Texcoco, los cuales sirven de soporte para los emprendedores que se acerquen al CDE.

Los $\mathrm{AE}$ son registrados generando un catálogo con su información de contacto personal, como su nombre, forma de contacto, dirección de correo electrónico, teléfono de contacto, y algún otro dato que este quiera brindar.

\subsection{Categorías de conocimientos en el CDE}

Debido a que los emprendedores que se acerquen al CDE, vendrán con la idea de un negocio, o la mejora del mismo; se debe tener una Base de Datos en la cual se cuente con un amplio repertorio de los conocimientos que tiene a su alcance el CDE.

Para que el usuario, que será el responsable de atender a los emprendedores en el $\mathrm{CDE}$, no se le complique la toma de decisión al momento de elegir entre los temas de 
asesoramiento que tiene dicho centro de desarrollo, se ha hecho una categorización de conocimientos. En vez de poner la palabra "Ingeniería", como una categoría la cual abarca subtemas en los cuales se tiene en muchas ocasiones más especialistas; se optó por poner específicamente categorías como "desarrollador web", "administrador de una base de datos", "mercadotecnia", "asesor de impuestos", etc.

\subsection{Alcance geográfico del CDE}

Los CDE se encuentran, en los Centros Universitarios. Los emprendedores recurren a los CDE, porque tienen la seguridad de que cuentan con el personal necesario y experimentado para que los logre a cumplir sus propósitos.

El alcance principal, en donde se lanza este sistema, es únicamente en la zona de Texcoco y municipios aledaños. Lo anterior porque se considera la movilidad de los AE y de los emprendedores.

\subsection{Diseño de la arquitectura de la aplicación}

Se optó por adaptar una arquitectura en capas basada en la arquitectura de Piattini [14]. Se incluyen 5 capas desde la obtención de la información hasta la aplicación que interactúa con el usuario, Fig. 2.

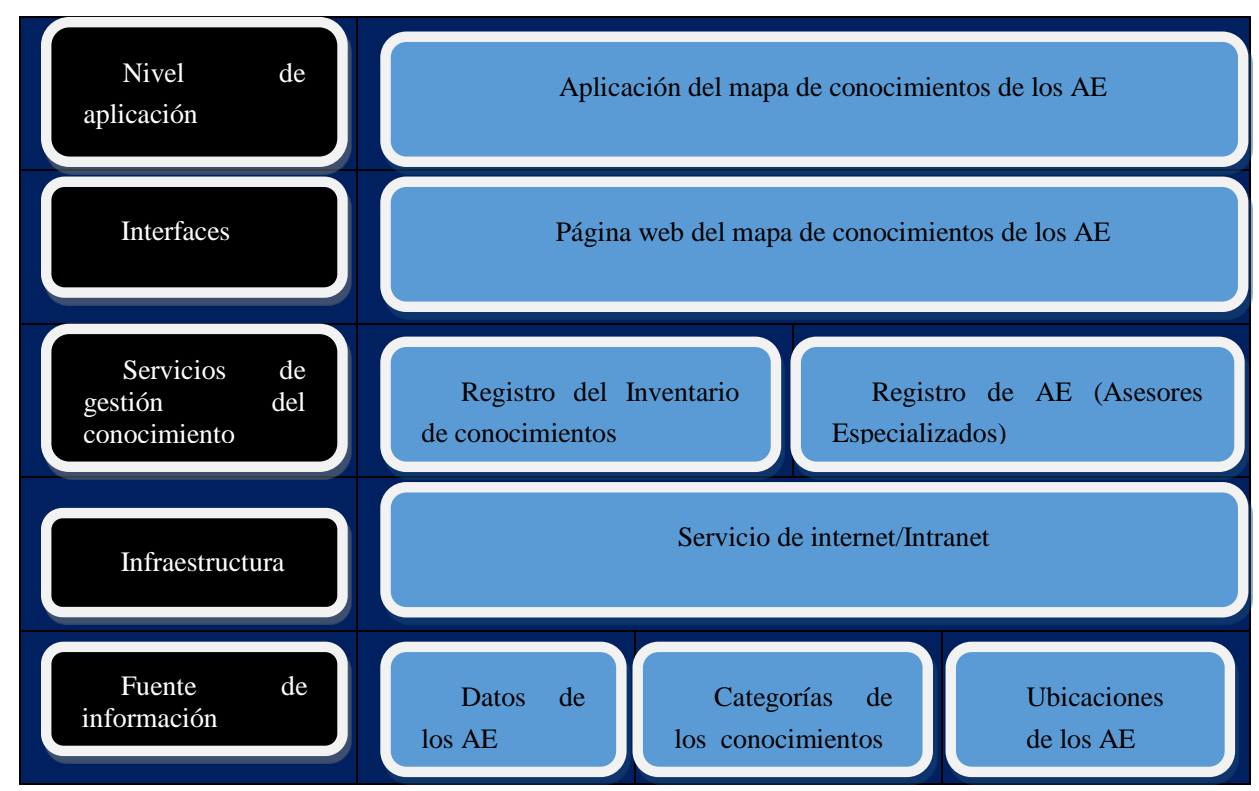

Fig. 2. Arquitectura de la aplicación del Mapa de Conocimientos (Adaptación de [14]).

Cabe precisar que no se trata de un SBC (Sistema Basado en el Conocimiento), sino más bien una plataforma que permite generar un mapa de conocimiento que apoya en la búsqueda del conocimiento y a los portadores. 


\subsection{Diseño de la Base de Datos}

En el diseño de la BD (Base de Datos), se usan tres tablas que son creadas desde la línea de comandos de MySQL Console ${ }^{8}$ con el apoyo de la suite de WampServer ${ }^{9}$, ya que dicha suite es gratuita y compatible con Windows, el sistema operativo que se está utilizando para este proyecto. La tabla 1se muestra a continuación:

Tabla 1. Tabla del catálogo de Asesores especializados.

\begin{tabular}{llllll}
\hline \multicolumn{2}{c}{ asesores_esp } & & & \\
\hline Id_ & Nombre_AE & Direccion_AE & Telefono & Correo & \multicolumn{1}{c}{ Id_ubic } \\
AE & & & & acion \\
1 & Ing. Antonio Diaz & $\begin{array}{l}\text { Plata s/n, Arenal 2, } \\
\text { Chicoloapan. }\end{array}$ & 5531494882 & ittonzs@gmail.com & 1 \\
& & CU UAEM Texcoco & 5531494882 & ittonzs@gmail.com & 2 \\
3 & Lic. Oscar Muro & CU UAEM Texcoco & 5531494882 & ittonzs@gmail.com & 3 \\
\hline
\end{tabular}

La tabla de Asesores Especializados, donde se incluirá la información de contacto de cada asesor, como su nombre, la dirección que el asesor quiera mostrar como contacto si es que tanto como el asesor y emprendedor requieran citas fueras del horario de operación de los $\mathrm{CDE}$, de igual forma el teléfono y dirección de correo electrónico, para poner en contacto al Emprendedor y al AE.

La tabla de Asesorías, permite alojar nombres de posibles asesorías la y clave de identificación de cada una. Se muestran los nombres de los AE en el sistema, y que el usuario podrá elegir para conocer quien posee el conocimiento de dicha asesoría. Es posible examinar la tabla 2 en seguida.

Tabla 2. Registro de asesorías.

\begin{tabular}{lll}
\hline \multicolumn{3}{c}{ Registro de Asesorías } \\
\hline Id_ase & Id_AE & Nombre_ase \\
\hline 1 & 1 & Base de Datos \\
2 & 2 & Desarrollo web \\
2 & 3 & Redes \\
\hline
\end{tabular}

En esta tabla se hacen las posibles combinaciones entre las Asesorías y Asesores, considerando que un Asesor puede poseer el conocimiento en dos o más temas a consultar.

La tabla 3 de Ubicaciones, es donde se establecen los lugares donde están los AE (podría ser más de uno), donde estén almacenadas las direcciones o ubicaciones.

8 Interfaz no gráfica de MySQL. MySQL es un sistema de gestión de bases de datos relacional lanzada por Sun Microsystems y actualmente por Oracle Inc.

9 Paquete de instalación que contiene un servidor (Apache), el gestor de base de datos MySQL y PHP como lenguaje de programación; para el Sistema Operativo Windows. 
API de Google Maps para un mapa de conocimiento de los asesores especializados ...

Tabla 3. Dirección o ubicación de los AE.

\begin{tabular}{lll}
\hline \multicolumn{3}{c}{ Direcciones de los AE } \\
\hline Id_ubicacion & latitud & longitud \\
\hline 1 & 19.417154 & 98.897545 \\
2 & 19.434422 & 98.916420 \\
3 & 19.434437 & 98.917503 \\
\hline
\end{tabular}

Estas ubicaciones están almacenadas con su latitud y longitud, puesto que la API de Google Maps usa las coordenadas para poder ubicar a nuestros AE en el mapa.

Para la obtención de dichas coordenadas, se requirió hacer la conversión de dirección física a coordenadas, lo que Google le llama Geocode Simple.

Pero gracias a la política de OpenSourceCode que Google maneja, se pueden encontrar aplicaciones que hacen esto, en donde se muestran los marcadores y al dar clic en alguno de ellos muestra las coordenadas necesarias para el sistema.

\subsection{Diseño de la interfaz del sistema}

Para el diseño de la interfaz se optó por la elaboración de una página web, para que en un futuro sea parte de la página principal de la institución.

Dicha interfaz es muy amigable con el usuario, solamente se tiene que elegir entre las distintas asesorías que cuenta el $\mathrm{CDE}$, para saber dónde y quién posee dicho conocimiento que le será de ayuda al emprendedor. La interfaz se puede apreciar en las vistas en las Fig. 2, 3 y 4 de la sección de resultados.

Para hacer uso del mapa de Google, ofrece su API la cual es una línea de código que se incrusta dentro del <head> (cabecera) de nuestro sitio. La versión más reciente de la API de Google Maps es la versión 3, la cual y está disponible en la página oficial de Google.

\subsection{Programación requerida}

Para la construcción del sitio web (únicamente la interfaz), se hizo usando HTML5 ${ }^{10}$, la versión de HTML más reciente, ayuda a la compatibilidad con los navegadores que existen actualmente.

De igual manera, como otros sitios web, se hacen utilizando JavaScript ${ }^{11}$, un lenguaje de programación para páginas y sitios web. Las sentencias que se usan son parecidas a programar en java, diferenciando un poco aquí pues se usan unas "etiquetas" para identificar el comienzo y final de una "instrucción". Por ejemplo para incluir la API de Google Maps se usa la etiqueta <script> en la que se declara la API, la cual manda a llamar a los servidores de Google que permita hacer uso del

${ }^{10}$ Es la versión 5 del lenguaje básico de la world wide web. Bajo la regulación del consorcio W3C.

11 Lenguaje de programación interpretado orientado a objetos. Se incrusta en el código HTML para que los sitios sean dinámicos. 
mapa, que cabe mencionar, que Google nos permite modificar y hacer sus mapas hasta cierto punto a nuestras necesidades, pues cuenta con varias opciones para adecuarse a nuestros proyectos; como pueden ser los markers, opciones del zoom, la ubicación para mostrar en primera instancia el mapa, por ejemplo nuestro sitio posicionarlo en un área donde quede centrada la zona de Texcoco, Estado de México. En el código siguiente se establece el mapa a mostrar.

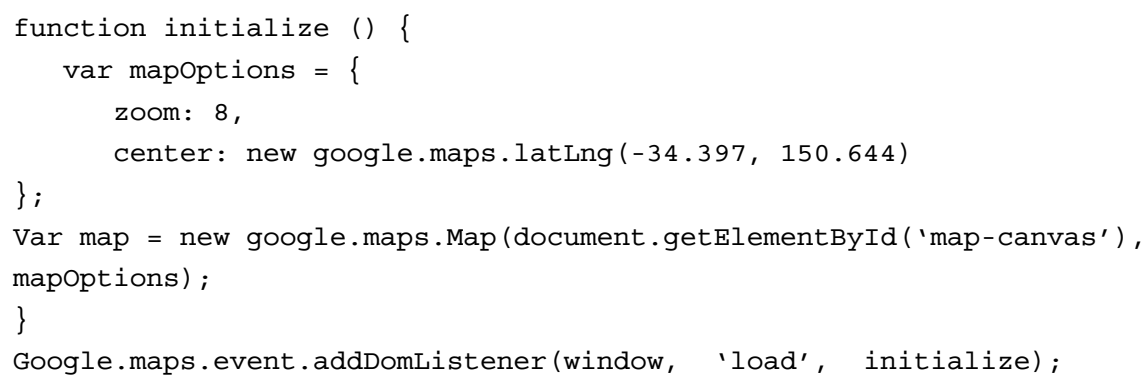

Dentro de la función principal se declara una variable, que es "mapOptions", la cual tiene dos opciones:

Zoom: nos permite mostrar el nivel de acercamiento visto desde el cielo para ver cierta zona del mapa.

Center: con esta opción nosotros le indicamos al mapa en que zona del mapa queremos posicionarnos a través de unas coordenadas de latitud y longitud.

Como el sistema que se desarrolló hace una consulta a través del combolist este debe de enviar dichas consultas a la base de datos y así muestra el resultado, pues lo que se hace es usar el método "post", que nos permite enviar la selección de nuestra consulta y arrojar un resultado sin que el usuario lo pueda ver, como usar el método "get", con el que se muestran todos los resultados al usuario y que para el sitio no es el caso.

Para usar el método "post" se declara en otro archivo, donde también se tiene que incluir el anterior archivo, al que se le ha llamado "puntosDados.php", y se incluye en el archivo de la misma manera que se incluyó el archivo de conexión de la BD. Se declaró a "post" como una variable global " \$_POST", para que la podamos usar en cualquier momento y con cualquier función sin tener que declararla para cada una de ellas.

Para ello en el archivo que se llamará "index.html", el cual es el principal para que pueda correr en un navegador, puesto que los navegadores buscan este nombre para poder mostrar algo en pantalla. Este archivo es donde se había declarado el “ $<$ !DOCTYPE hmtl $>$ " y donde estaba el " $<$ script $>$ " de la API de Google Maps [8].

\subsection{GEOCODE SIMPLE (Codificación Geográfica)}

Es el proceso de transformar direcciones (como "1600 Amphitheatre Parkway, Mountain View, CA") en coordenadas geográficas (como 37.423021 de latitud y 122.083739 de longitud), que se pueden utilizar para colocar marcadores o situar el mapa. El API de codificación geográfica de Google proporciona una forma directa de 
acceder a un geocoder mediante solicitudes HTTP. Además, el servicio permite realizar la operación contraria (convertir coordenadas en direcciones); este proceso se conoce con el nombre de "codificación geográfica inversa".

\section{Resultados}

El resultado obtenido de este trabajo consiste en una aplicación web capaz de funcionar en dispositivos con un navegador y conexión a internet. Un administrador del sistema cargó un catálogo de categorías de conocimiento de acuerdo al Inventario de Conocimientos que tiene el CDE a través de los AE. También fue necesario registrar los $\mathrm{AE}$ con sus datos de ubicación y formas de contacto. Para cada AE se debió determinar su ubicación geográfica para poder mapear su existencia. La aplicación filtra los AE de acuerdo a la categoría elegida, y solamente muestra los $\mathrm{AE}$ existentes. Cuando se elige una categoría y no se tienen ningún experto, no se muestra ninguna marca. La página principal se puede observar en la figura 3.

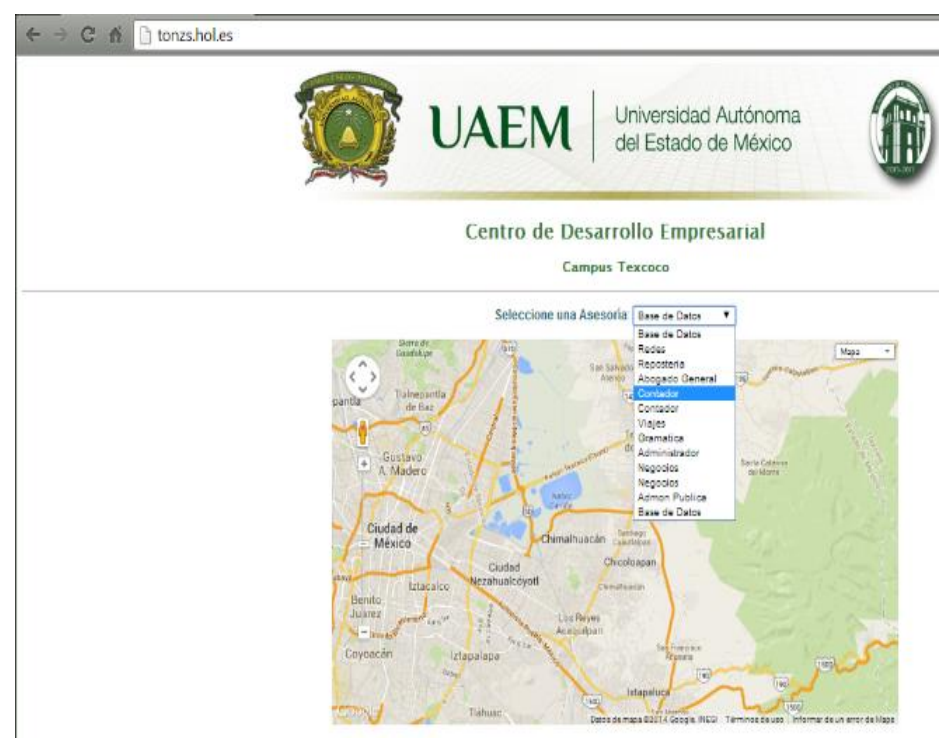

Fig. 3. Vista de la aplicación para la elección de categoría de conocimiento.

Una vez que se elige una categoría de conocimiento, la aplicación filtra los AE que poseen ese tipo de conocimiento y se muestran marcas en el mapa.

El usuario puede dar un clic en la marca para desplegar los datos del AE, como se muestra en la Fig. 5. El sistema no emite automáticamente el aviso a los AE, pero es posible, agregar la función; por ahora toma los datos de contacto y podrá de forma tradicional contactar y acordar detalles de la asesoría requerida.

La aplicación se ha implementado en un servidor del CDE del Centro Universitario UAEM Texcoco, se están registrando el inventario de conocimiento y los AE, posteriormente se valorará su impacto. 


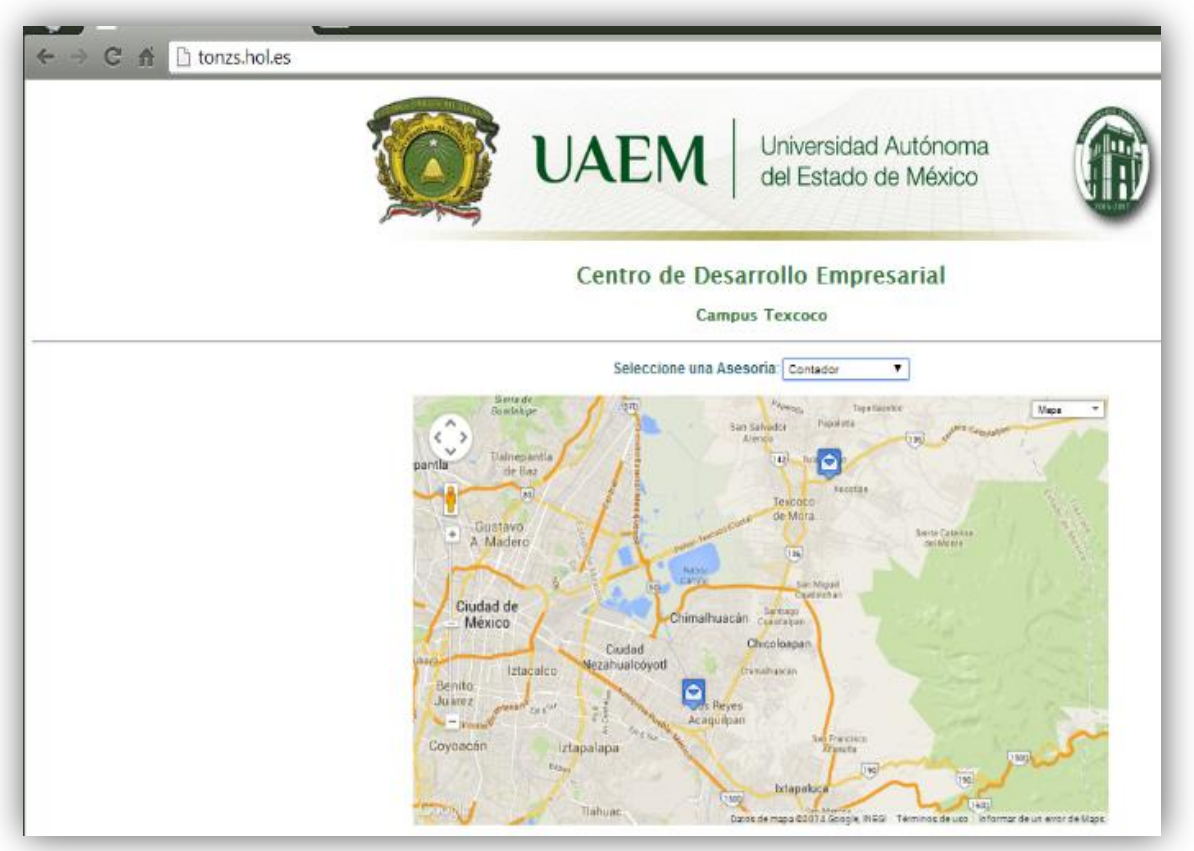

Fig. 4. Marcas de AE que poseen el conocimiento elegido.

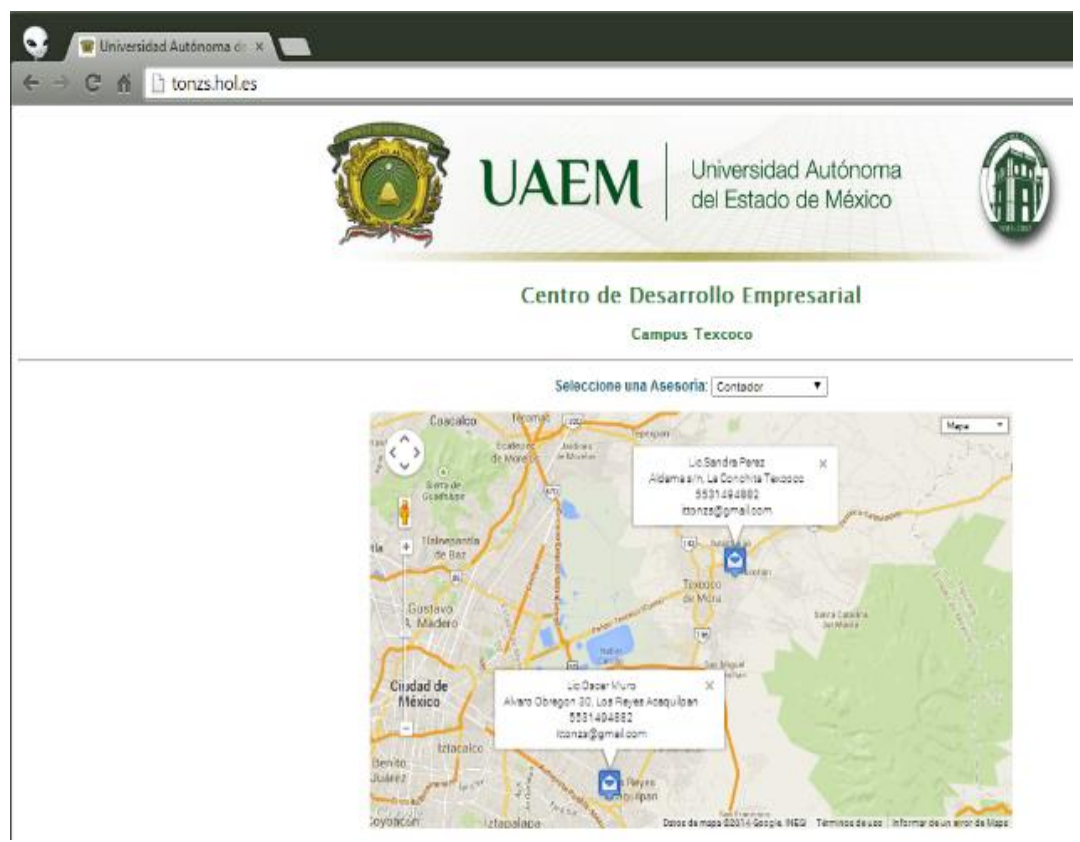

Fig. 5. Mapa con los datos de los AE desplegados. 


\section{Conclusiones y trabajos futuros}

Después de haber llevado a cabo pruebas con diferentes navegadores, se observó que no existe variación alguna en cuanto al comportamiento de los elementos del sitio web, así como tampoco en los resultados que arrojaron las consultas hechas, y esto se debe a que desde que se empezó a programar y diseñar el sitio, se contempló utilizar las sentencias que indicaban ser las versiones más recientes y estables de HTML como de la API de Google Maps.

Desde una primera instancia se tenía como objetivo que el sistema funcionara arrojando los resultados de las consultas en un mapa. Al final la aplicación cumple el cometido y se logra el objetivo principal.

La aplicación resuelve el problema del CDE porque permite que los usuarios accedan a la aplicación elijan una categoría de conocimiento existente y obtengan un mapa que muestra la existencia de conocimiento; así como la ubicación y a los portadores.

Durante la implementación fue fácil y amigable; además de encontrar la gran gama de posibilidades para otras soluciones para mapear no solo conocimiento sino otros aspectos; como; seguridad, riesgos, mercados, etc.

Se hicieron pruebas por parte de usuarios, personal del CDE y fuera de la región de Texcoco arrojando los resultados esperados.

Se planean dos trabajos futuros: El primero consiste en lograr que el sistema permita la programación, registro, monitoreo y estadísticas de las asesorías a los emprendedores y empresarios por parte de los AE. La segunda consiste en agregar información más específica del conocimiento, AE y ubicación con el fin de agregar un algoritmo genético para determinar cuál es el AE que más conviene contactar.

\section{Referencias}

1. Carrión, J.: Gestión del conocimiento. Recuperado en Mayo, 2002, vol. 4, p. 2007.

2. Centro Universitario UAEM Texcoco. Centro de Desarrollo Empresarial. http://incemtex.mx/

3. Centro Universitario UAEM Texcoco. Centro de Desarrollo Empresarial.

4. Collison, C., Parcell, G.: La gestión del conocimiento, Lecciones prácticas de una empresa líder. Buenos Aires: Paidós (2003)

5. Davenport, T., Prusak, L.: Conocimiento en acción, Como las organizaciones manejan lo que saben. Buenos Aires: Prentice Hall (2001)

6. Davenport, T., et al.: Building Successful Knowledge Management Projects. [En línea]. 16-04-13, Disponible en Internet: http://www.providersedge.com/docs/km_articles/Building_Successful_KM_Projects.pdf

7. De la Espriella Fortoul, L.M, Pineda Pinzon, D.C.: Gestión del conocimiento. Soluciones efectivas S.A. Articulo. [en línea][23-04-13]. Disponible en internet: http://hdl.handle.net/10818/2012

8. Google Maps. Obtenido el 23 de abril de 2013 de https://developers.google.com/maps/web/

9. http://incemtex.mx/ 
10. Martínez, Martínez, A., \& Corrales, M. (2010). Administración de conocimiento y desarrollo basado en conocimiento, redes e innovación. México D. F.: CENGAGE Learning.

11. Nonaka, S., \& Takeuchi, N. (1999). La organización creadora del conocimiento. México: Oxford.

12. Normalización y Certificación Electrónica A. C. (2007). Guía práctica de implantación de los requisitos de la NMX-I-059-NYCE-2005 (MoPRoSoft). México D. F.: Normalización y Certificación Electrónica, A. C.

13. NYCE A. C:. (2007). Guía práctica de implantación de los requisitos de la NMX-I-059NYCE-2005 (MoProSoft) (Primera ed.). México D. F.: Normalización y Certificación A. C.

14. Piattini, M.; García, F. y Caballero, I. Calidad de Sistemas Informáticos. México, DF: Alfa Omega Grupo Editor, S.A de C. V., 2007. 416p. ISBN: 978-970-15-1267-8.

Ruiz, S., Ledeneva, Y., \& Morales, R. (2012). Base de conocimiento de los procesos de desarrollo de software a través de un modelo de un sistema de gestión del conocimiento. Research in Computing Science, Avances en inteligencia artificial, 55, 113-123. 